\title{
Many-Body Delocalization in the Presence of a Quantum Bath
}

\author{
Antonio Rubio-Abadal, ${ }^{1, *}$ Jae-yoon Choi, ${ }^{1,2,{ }^{*}, \dagger}$ Johannes Zeiher, ${ }^{1}$ Simon Hollerith, ${ }^{1}$ Jun Rui, ${ }^{1}$ \\ Immanuel Bloch, ${ }^{1,3}$ and Christian Gross $\oplus^{1}$ \\ ${ }^{1}$ Max-Planck-Institut für Quantenoptik, 85748 Garching, Germany \\ ${ }^{2}$ Department of Physics, Korea Advanced Institute of Science and Technology, Daejeon 3414, Korea \\ ${ }^{3}$ Fakultät für Physik, Ludwig-Maximilians-Universität München, 80799 München, Germany
}

(Received 30 April 2018; revised manuscript received 26 September 2018; published 18 October 2019)

\begin{abstract}
Closed generic quantum many-body systems may fail to thermalize under certain conditions even after long times, a phenomenon called many-body localization (MBL). Numerous studies support the stability of the MBL phase in strongly disordered one-dimensional systems. However, the situation is much less clear when a small part of the system is ergodic, a scenario which also has important implications for the existence of many-body localization in higher dimensions. Here we address this question experimentally using a largescale quantum simulator of ultracold bosons in a two-dimensional optical lattice. We prepare two-component mixtures of varying relative population and implement a disorder potential which is experienced only by one of the components. The second nondisordered "clean" component plays the role of a bath of adjustable size that is collisionally coupled to the "dirty" component. Our experiments show how the dynamics of the dirty component, which, when on its own, show strong evidence of localization, become affected by the coupling to the clean component. For a high clean population, the clean component appears to behave as an effective bath for the system which leads to its delocalization, while for a smaller clean population, the ability of the bath to destabilize the system becomes strongly reduced. Our results reveal how a finite-sized quantum system can bring another one towards thermalization, in a regime of complex interplay between disorder, tunneling, and intercomponent interactions. They provide a new benchmark for effective theories aiming to capture the complex physics of MBL in the weakly localized regime.
\end{abstract}

DOI: 10.1103/PhysRevX.9.041014

\section{INTRODUCTION}

Typical quantum many-body systems evolve into a locally thermal state after driven out of equilibrium by a global quench [1]. This quantum version of thermalization is explained by the eigenstate thermalization hypothesis, which postulates that small subsystems are described by a thermal density matrix even for individual many-body eigenstates of the global system [2-5]. Quantum thermalization can, however, fail generically in systems exhibiting quenched disorder [6-8] when the strength of the disorder is large enough to prevent efficient spreading of entanglement [9]. Nonthermalizing behavior and strong indication for the existence of a many-body-localized (MBL) phase have been observed experimentally in several systems, in one [10-14] as well as in two dimensions [15,16]. An efficient delocalization of such MBL systems can be

\footnotetext{
*These authors contributed equally to this work.

†jaeyoon.choi@kaist.ac.kr
}

Published by the American Physical Society under the terms of the Creative Commons Attribution 4.0 International license. Further distribution of this work must maintain attribution to the author(s) and the published article's title, journal citation, and DOI.
Subject Areas: Atomic and Molecular Physics, Condensed Matter Physics

induced by coupling them to an external dissipative bath, which will ultimately restore thermalization. But similar processes can take place even in perfectly isolated systems, when a large enough part of the system is ergodic. Specifically, the many-body eigenstates in some parts of the energy spectrum may obey the eigenstate thermalization hypothesis while in other parts remain localized [17-19] and spatial rare regions of low disorder may form local ergodic inclusions $[20,21]$ that can trigger a destabilizing avalanche, a common argument against the existence of a MBL phase in higher dimensions. An understanding of the mechanisms which lead to thermalization and to the destruction of MBL is thus of central importance to verify the robustness of the MBL phase. Such processes pose a challenge for both numerical and experimental methods, since their identification requires the study of long evolution times in large quantum systems.

An experiment which allows us to probe such delocalizing phenomena in a highly controlled setting and in the complex regime near the transition between the ergodic and localized phases consists of an interacting two-component mixture composed of a "dirty" component in a random potential and a "clean" component insensitive to the disorder [22-24]. In such a hybrid system, the clean component, which on its own would quantum thermalize, can be 
viewed as a quantum bath with a tunable number of degrees of freedom [25-27]. In particular, the small-bath regime can be realized, a very different scenario compared to the theoretically [28-31] and experimentally studied [32] coupling of a MBL system to a classical bath at infinite coupling bandwidth. Under some conditions, a small quantum bath might even fail to thermalize the whole system. Such a breakdown of thermalization can happen as a consequence of the bath becoming localized via the intercomponent interactions, which can play the role of an effective disorder (a MBL proximity effect $[22,23]$ ). In this work, we use a quantum-gas microscope to prepare an out-of-equilibrium state in a disordered potential and to measure its dynamics for long timescales beyond 1000 tunneling times. By introducing a second species insensitive to the disorder, we realize the setting described above. While the dirty component shows strong indication of localization in the absence of a bath, the introduction of a large enough number of clean atoms alters the dynamics qualitatively, and the signs of localization vanish eventually.

\section{EXPERIMENTAL SETUP}

Our experimental system consists of a square optical lattice with lattice spacing $a_{\mathrm{lat}}=532 \mathrm{~nm}$ in which we load an ultracold cloud of ${ }^{87} \mathrm{Rb}$ atoms. By preparing the

(a)
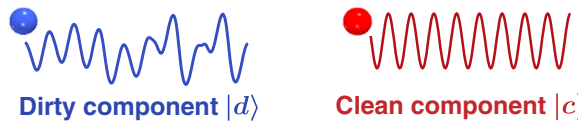

Clean component $|c\rangle$

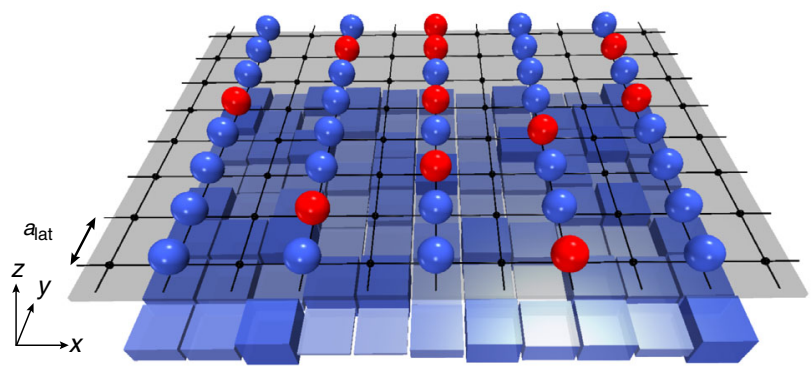

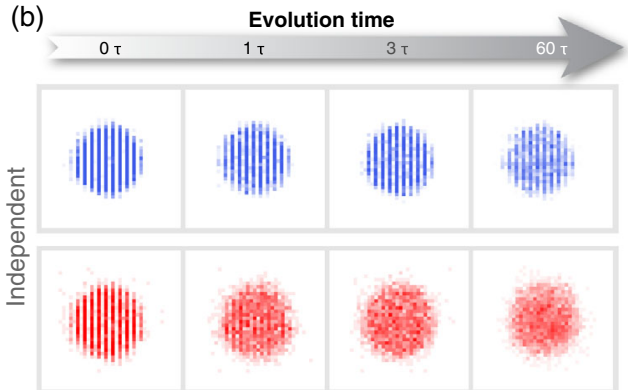

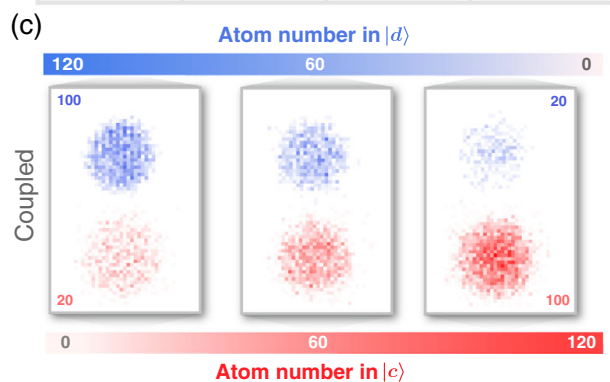

FIG. 1. Schematic description of the experiment. (a) Illustration of the two-component mixture at the beginning of the experimental sequence. The system consists of a square optical lattice with species-dependent disorder in which two different bosonic hyperfine spin states are prepared. The clean component (red, $|c\rangle$ ) experiences only the lattice potential, while the dirty component (blue, $|d\rangle$ ) is additionally affected by a random on-site potential (blue boxes of different lightness). The initial state is prepared with a short-scale density modulation along the $x$ direction. (b) Dynamics of the mean density distribution for systems prepared independently with only either one of the two components. The dirty component partially preserves the initial-state density modulation for a sufficiently high disorder strength (in blue, for $\Delta=28 J$ ), which is a signature of the breakdown of ergodicity and the formation of a MBL phase. The clean component $|c\rangle$, in contrast, relaxes in a few tunneling times to a state with no clear density modulation (in red). (c) Mean stateselective density distribution of a mixture after a long evolution time. The measurements are taken after $281 \tau$ evolution for three exemplary component fractions. 
second column such that $N=124(12)$ atoms remain [see Fig. 1(b)]. We then prepare a fraction of the atoms in state $|c\rangle$ via a microwave pulse before imposing the disorder potential (see Fig. 1) and quenching the optical-lattice tunneling down to $J / \hbar=2 \pi \times 24.8 \mathrm{~Hz}$ and the interactions to $U=24.4 \mathrm{~J}$. The random disorder potential is chosen to be different for each experimental realization, and its distribution is approximately Gaussian with a full width at half maximum of $\Delta=28 J$ [15]. The described procedure involving a simultaneously quench of lattice depth, disorder strength, and filling of the system leads to a prepared state with a high energy density in the many-body spectrum of the system (see a more detailed discussion in the Supplemental Material [34]). After the lattice quench, we allow the system to evolve for up to $t \simeq 1100 \tau$, where $\tau=\hbar / J$ is the characteristic tunneling time. After the evolution, the lattices are increased to their maximum depth in order to freeze the spatial distribution and image the atomic occupations on each individual lattice site [35]. For the chosen value of the disorder strength $\Delta$, the density distribution of the dirty component eventually reaches a steady state, retaining a signature of the initial density modulation, while the clean one quickly becomes featureless [see Fig. 1(b)]. The access to the individual site occupations allows us to characterize these dynamics by tracking the imbalance $\mathcal{I}=\left(N_{e}-N_{o}\right) /\left(N_{e}+N_{o}\right)$, where $N_{e, o}$ are the occupation numbers on sites at even and odd columns. The use of the short-distance density modulation efficiently probes localization at short length scales.

\section{DYNAMICS WITHOUT BATH}

We start by preparing a state in which all particles are in the dirty component, i.e., in the absence of the atomic bath, and measure the evolution of its imbalance $\mathcal{I}_{d}$. Over a few hundreds of tunneling times, we observe a decrease of the initial imbalance from $\mathcal{I}_{d}=0.91(1)$ to a long-time quasisteady value of $\mathcal{I}_{d} \approx 0.13$ - a signature of MBL. The measured dynamics can be phenomenologically well described by the sum of two exponentials with vastly different time constants and a stationary offset. During the first identified period characterized by a decay time of $0.6(1) \tau$, the atoms expand freely into empty sites. In the following period of much slower dynamics [decay time $103(6) \tau]$, interactions are important and, together with the even longer time dynamics, this timescale constitutes the focus of our analysis. In addition, we resolve the formation of doubly occupied sites (doublons) during the relaxation dynamics, starting from an initially doublon-free case, which exhibits a rapid growth and subsequent saturation after the quench. This effect requires both interactions and disorder, since in a disorder-free lattice with the same parameters, such a dynamical doublon formation is strongly suppressed by the interactions. The qualitative behavior of these dynamics is reproduced by small-system numerical simulations (see Supplemental Material [34]).
Furthermore, recent numerical work on the 2D disordered Bose-Hubbard model has found signatures of MBL by studying individual eigenstate properties at parameters consistent with this work [36]. Nonetheless, the finite coupling to the environment in our system, inevitable in any experiment, becomes increasingly important at longer times. Its dominant effect is an atom loss of $15 \%$ after $600 \tau$. The effect of such a loss on localization is not entirely clear, but in our experiments it does not seem to cause strong delocalization. No total relaxation of the imbalance has been observed even for the longest measured times, which are among the longest times probed in any MBL-related experiment so far. The data of Fig. 2 show that the timescale of any potential subsequent relaxation is large and well separated from the characterized initial decay. In addition, an exponential fit of the data after $500 \tau$, combined with a bootstrap analysis, allows us to bound any further relaxation to be $t_{3}>2300 \tau$ with $92 \%$ confidence. This lower bound is our sensitivity limit for the relaxation, in the sense that any slower decay process cannot be distinguished from true localization. We define this long-time behavior as a "quasisteady state." The separation of timescales between the initial decay and any potential further decay is,

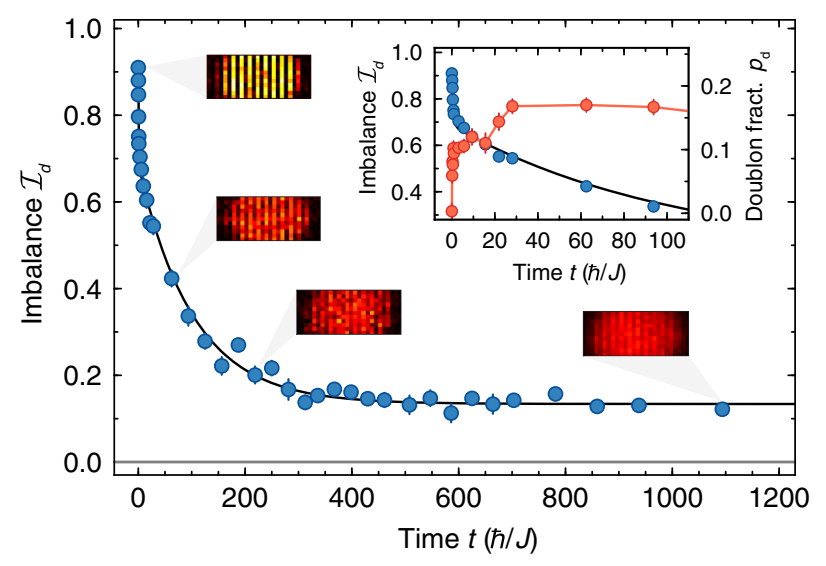

FIG. 2. Dynamics of the dirty component alone. Evolution of the imbalance $\mathcal{I}_{d}$ (points) for a system composed only of the dirty component $|d\rangle$. The blue points show the measured data and the solid line a fit of the points to the sum of two exponentials including an offset. The imbalance decreases monotonically, albeit on distinct timescales. An initial decay, where interactions are negligible, is followed by a slower second timescale governed by doublon relaxation. After approximately $300 \tau$, a quasisteady state of finite imbalance $\mathcal{I}_{d} \approx 0.13$ is reached. The four rectangular boxes show the averaged density distribution in the trap center (black-red-yellow color scale) at times $0 \tau, 63 \tau, 219 \tau$, and $1094 \tau$, displaying the reduction in the imbalance. In the inset, we show the short-time dynamics of the imbalance $\mathcal{I}_{d}$ (blue) and the doublon population $p_{\mathrm{d}}$ (red) defined as the fraction of atoms in doubly occupied lattice sites. Notably, the doublon formation rate changes markedly between the two dynamical regimes of the imbalance decay. The error bars represent 1 standard deviation of the mean. 
however, large, rendering the observed plateau a useful indication of the presence of MBL in the system.

\section{DYNAMICS WITH BATH}

We now turn to studying the effects of coupling a tunable atomic bath to the system. To this end, we initially prepare a mixture with a preset number $N_{c}$ of atoms in the clean state, and after the dynamics, we ensure that the detection is only sensitive to atoms in state $|d\rangle$ by removing all $|c\rangle$ atoms with a resonant light pulse prior to detection (see Supplemental Material [34]). The evolution of the imbalance $\mathcal{I}_{d}$ for three different bath sizes $\left(N_{c}=20,40,90\right)$ is shown in Fig. 3(a), together with the dirty-only case as reference. Generally, the larger the bath, the smaller the imbalance in the long-time limit. While introducing a fraction of clean atoms also implies reducing the density of the dirty component, it is important to consider that this alone would actually yield a higher long-time imbalance [15]. In the $N_{c}=90$ case, the imbalance relaxes to a vanishing value in less than $300 \tau$, implying that the particles have delocalized over at least several lattice sites. A time constant of $140(30) \tau$ can be extracted from a single exponential fit (red dashed line) for this relaxation process. A similar delocalization takes place for the $N_{c}=40$ case, whose exponential fit gives a slower time constant of $200(20) \tau$. These results indicate that the clean component indeed acts as an effective bath, destabilizing and thermalizing the localized dirty component. Importantly, this effect is caused by collisional interactions only, whose strength

(a)

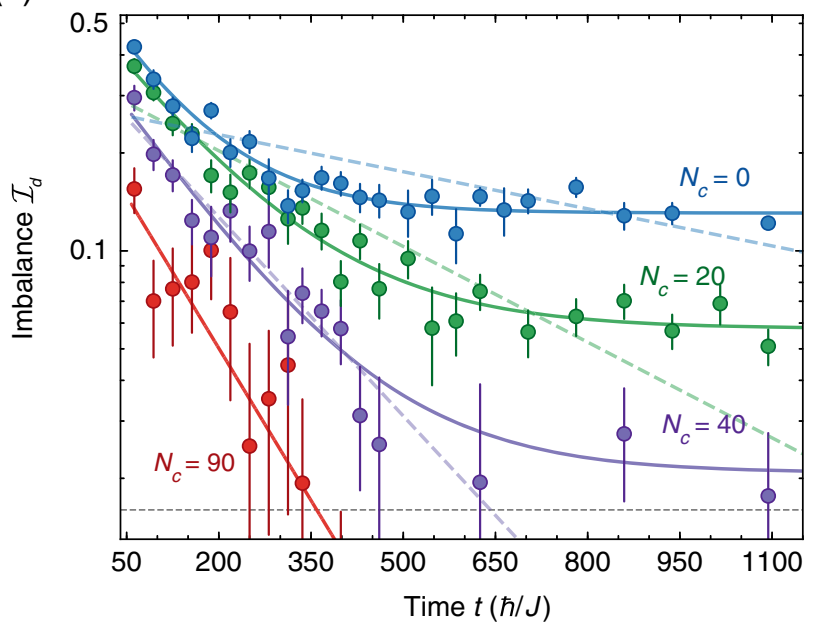

is equal to the intracomponent interactions in the system; i.e., no new energy scale is introduced when adding the clean component. When reducing the size of the bath even further, to $N_{c}=20$, we still observe an overall reduction of the imbalance, but the dynamics is qualitatively different. A finite imbalance still persists for the longest measured times, and a simple exponential fit does not describe the dynamics accurately anymore. Introducing a steady-state offset, as in the case without a bath, matches the data significantly better (see Supplemental Material [34]), such that this is the simplest model describing all datasets (solid lines). A bootstrap analysis of the data after $625 \tau$ allows us to bound the subsequent relaxation to be $t_{3}>1100 \tau$ with a confidence of $92 \%$.

The observed dynamics for smaller baths may be explained by an inefficient delocalization of the dirty component, with a decay rate much smaller than the other timescales in the system but could also hint at a failure of thermalization. Theoretical studies for finite 1D systems with coupled clean and dirty components [22,23] found a persisting localization in certain regimes of reduced tunneling of the clean component, for which the coupling rate of spatially separated points is decreased. To quantify the delocalizing effect of the bath at long evolution time, we show the imbalance $\mathcal{I}_{d}$ as a function of the bath size [see Fig. 3(b)] for two different evolution times $(t=859 \tau$ and $t=1094 \tau)$. The values at the two times are similar, and they cannot be distinguished from the steady-state offsets obtained from the fits of Fig. 3(a). For bath sizes below $N_{c} \approx 40$, a finite imbalance $\mathcal{I}_{d}$ remains at long times.

(b)

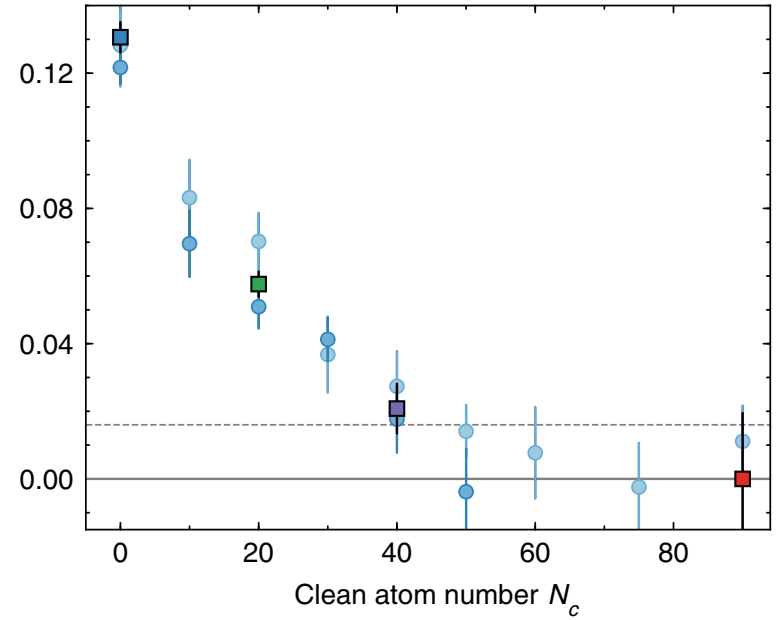

FIG. 3. Dynamics and delocalization in the presence of a quantum bath. (a) Dynamics of the dirty-state imbalance $\mathcal{I}_{d}$ for four different bath sizes $\left(N_{c}=0\right.$ in dark blue, $N_{c}=20$ in green, $N_{c}=40$ in purple, and $N_{c}=90$ in red). The dashed lines indicate exponential fits and the solid lines fits of an exponential with an offset. Introducing the clean component leads to delocalization indicated by the reduced imbalance. The imbalance relaxes completely for the two largest bath sizes, while for the smallest size of the bath $\left(N_{c}=20\right)$, a finite imbalance remains. (b) Experimental steady-state imbalance as a function of the bath size. The data are measured at $t=859 \tau$ (round points in blue) and at $t=1094 \tau$ (round points in light blue). The square points correspond to the asymptotic offsets obtained from the four solid line fits in (a). The horizontal dashed gray line indicates the typical statistical threshold at which the imbalance is compatible with zero. The error bars indicate 1 standard deviation of the mean. 


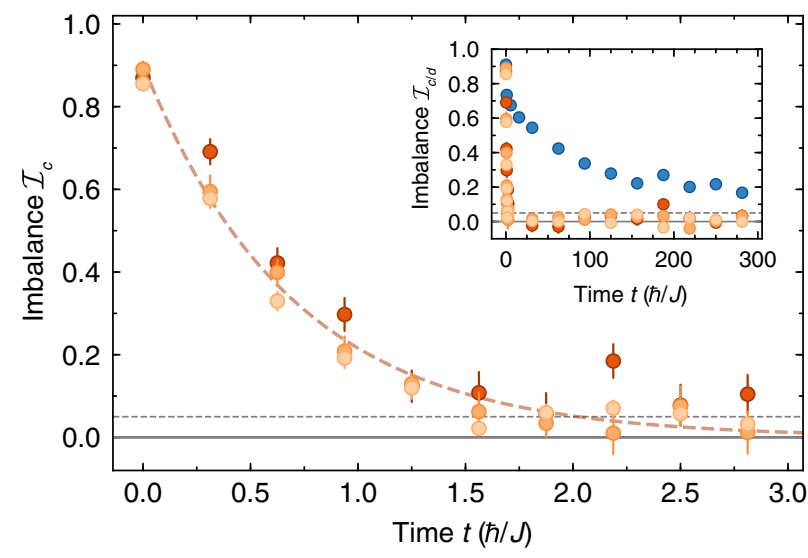

FIG. 4. Imbalance dynamics of the clean component. Evolution of the clean component imbalance $\mathcal{I}_{c}$ for three different atom numbers of the clean component $\left(N_{c}=120\right.$ in light orange, $N_{c}=60$ in orange, and $N_{c}=20$ in dark orange). The dashedline curve is an exponential fit with time constant of $0.7 \tau$. All bath sizes result in a similar behavior, with the imbalance vanishing in few tunneling times. The inset shows the long-time evolution of the clean component compared with the dynamics of the dirty component in the $N_{c}=0$ case, highlighting the strong difference in the timescales. The horizontal dashed gray lines indicate the typical statistical threshold at which the imbalance is compatible with zero. The error bars represent 1 standard deviation of the mean.

Finally, to probe the backaction of the dirty component on the bath dynamics, we also track the evolution of the bath component in our system. This is realized by removing the dirty component $|d\rangle$ prior to detection in order to detect the clean particles $|c\rangle$ only (Fig. 4). The results show that no matter how small the bath is, $\mathcal{I}_{c}$ relaxes very quickly, on a timescale of a few tunneling times $\tau$. Any potential interaction-induced localization of the small bath by the dirty component would therefore be characterized by a localization length spanning many sites, beyond what can be detected by the short-distance probing used here. Such a behavior is expected given that even the remaining imbalance of the dirty component $\mathcal{I}_{d}=0.07(2)$ in the $N_{c}=20$ case is already very small; i.e., it can serve only as a rather weak disorder source.

\section{CONCLUSION}

The experiments reported in this work shed first light on MBL systems in contact with a quantum bath of tunable size. Generally, the presence of such a bath tends to drive the system towards delocalization, which eventually becomes complete even on our experimentally accessible timescales, when the bath formed by the clean component becomes large enough. Follow-up experiments may explore different initial states and disorder regimes to settle the question of proximity-induced localization [22,23], demonstrating the localization of a system due to and not despite interactions. Furthermore, the debated question on the stability of MBL in the presence of thermal inclusions can be directly addressed in a similar experiment with engineered low-disorder regions [20,21].

\section{ACKNOWLEDGMENTS}

We acknowledge fruitful discussions with D. A. Huse, P. Bordia, H. Lüschen, Y. Bar Lev, and M. Heyl. Financial support is provided by the Max Planck Society and the European Union Marie Curie Fellowship to J.-y. C.

A. R.-A. and J.-y. C. contributed equally to this work.

[1] M. Rigol, V. Dunjko, and M. Olshanii, Thermalization and Its Mechanism for Generic Isolated Quantum Systems, Nature (London) 452, 854 (2008).

[2] J. M. Deutsch, Quantum Statistical Mechanics in a Closed System, Phys. Rev. A 43, 2046 (1991).

[3] M. Srednicki, Chaos and Quantum Thermalization, Phys. Rev. E 50, 888 (1994).

[4] H. Tasaki, From Quantum Dynamics to the Canonical Distribution: General Picture and a Rigorous Example, Phys. Rev. Lett. 80, 1373 (1998).

[5] A. M. Kaufman, M. E. Tai, A. Lukin, M. Rispoli, R. Schittko, P. M. Preiss, and M. Greiner, Quantum Thermalization through Entanglement in an Isolated Many-Body System, Science 353, 794 (2016).

[6] P. W. Anderson, Absence of Diffusion in Certain Random Lattices, Phys. Rev. 109, 1492 (1958).

[7] D. Basko, I. Aleiner, and B. Altshuler, Metal-Insulator Transition in a Weakly Interacting Many-Electron System with Localized Single-Particle States, Ann. Phys. (Amsterdam) 321, 1126 (2006).

[8] I. V. Gornyi, A. D. Mirlin, and D. G. Polyakov, Interacting Electrons in Disordered Wires: Anderson Localization and Low-T Transport, Phys. Rev. Lett. 95, 206603 (2005).

[9] R. Nandkishore and D. A. Huse, Many-Body Localization and Thermalization in Quantum Statistical Mechanics, Annu. Rev. Condens. Matter Phys. 6, 15 (2015).

[10] M. Schreiber, S. S. Hodgman, P. Bordia, H. P. Lüschen, M. H. Fischer, R. Vosk, E. Altman, U. Schneider, and I. Bloch, Observation of Many-Body Localization of Interacting Fermions in a Quasirandom Optical Lattice, Science 349, 842 (2015).

[11] J. Smith, A. Lee, P. Richerme, B. Neyenhuis, P. W. Hess, P. Hauke, M. Heyl, D. A. Huse, and C. Monroe, Many-Body Localization in a Quantum Simulator with Programmable Random Disorder, Nat. Phys. 12, 907 (2016).

[12] P. Roushan et al., Spectroscopic Signatures of Localization with Interacting Photons in Superconducting Qubits, Science 358, 1175 (2017).

[13] K. X. Wei, C. Ramanathan, and P. Cappellaro, Exploring Localization in Nuclear Spin Chains, Phys. Rev. Lett. 120, 070501 (2018).

[14] K. Xu, J.-J. Chen, Y. Zeng, Y.-R. Zhang, C. Song, W. Liu, Q. Guo, P. Zhang, D. Xu, H. Deng, K. Huang, H. Wang, X. Zhu, D. Zheng, and H. Fan, Emulating Many-Body 
Localization with a Superconducting Quantum Processor, Phys. Rev. Lett. 120, 050507 (2018).

[15] J.-y. Choi, S. Hild, J. Zeiher, P. Schauß, A. Rubio-Abadal, T. Yefsah, V. Khemani, D. A. Huse, I. Bloch, and C. Gross, Exploring the Many-Body Localization Transition in Two Dimensions, Science 352, 1547 (2016).

[16] P. Bordia, H. Lüschen, S. Scherg, S. Gopalakrishnan, M. Knap, U. Schneider, and I. Bloch, Probing Slow Relaxation and Many-Body Localization in TwoDimensional Quasiperiodic Systems, Phys. Rev. X 7, 041047 (2017).

[17] X. Li, S. Ganeshan, J. H. Pixley, and S. D. Sarma, Many-Body Localization and Quantum Nonergodicity in a Model with a Single-Particle Mobility Edge, Phys. Rev. Lett. 115, 186601 (2015).

[18] W. De Roeck, F. Huveneers, M. Müller, and M. Schiulaz, Absence of Many-Body Mobility Edges, Phys. Rev. B 93, 014203 (2016).

[19] H. P. Lüschen, S. Scherg, T. Kohlert, M. Schreiber, P. Bordia, X. Li, S. D. Sarma, and I. Bloch, Exploring the SingleParticle Mobility Edge in a One-Dimensional Quasiperiodic Optical Lattice, Phys. Rev. Lett. 120, 160404 (2018).

[20] K. Agarwal, E. Altman, E. Demler, S. Gopalakrishnan, D. A. Huse, and M. Knap, Rare-Region Effects and Dynamics near the Many-Body Localization Transition, Ann. Phys. (Berlin) 529, 1600326 (2017).

[21] W. De Roeck and J.Z. Imbrie, Many-Body Localization: Stability and Instability, Phil. Trans. R. Soc. A 375, 20160422 (2017).

[22] R. Nandkishore, Many-Body Localization Proximity Effect, Phys. Rev. B 92, 245141 (2015).

[23] K. Hyatt, J. R. Garrison, A. C. Potter, and B. Bauer, Many-Body Localization in the Presence of a Small Bath, Phys. Rev. B 95, 035132 (2017).

[24] J. Marino and R. M. Nandkishore, Many-Body Localization Proximity Effects in Platforms of Coupled Spins and Bosons, Phys. Rev. B 97, 054201 (2018).

[25] D. A. Huse, R. Nandkishore, F. Pietracaprina, V. Ros, and A. Scardicchio, Localized Systems Coupled to Small Baths: From Anderson to Zeno, Phys. Rev. B 92, 014203 (2015).
[26] S. Banerjee and E. Altman, Variable-Range Hopping through Marginally Localized Phonons, Phys. Rev. Lett. 116, 116601 (2016).

[27] P. Bordia, H. P. Lüschen, S. S. Hodgman, M. Schreiber, I. Bloch, and U. Schneider, Coupling Identical OneDimensional Many-Body Localized Systems, Phys. Rev. Lett. 116, 140401 (2016).

[28] R. Nandkishore, S. Gopalakrishnan, and D. A. Huse, Spectral Features of a Many-Body-Localized System Weakly Coupled to a Bath, Phys. Rev. B 90, 064203 (2014).

[29] S. Johri, R. Nandkishore, and R. N. Bhatt, Many-Body Localization in Imperfectly Isolated Quantum Systems, Phys. Rev. Lett. 114, 117401 (2015).

[30] E. Levi, M. Heyl, I. Lesanovsky, and J. P. Garrahan, Robustness of Many-Body Localization in the Presence of Dissipation, Phys. Rev. Lett. 116, 237203 (2016).

[31] M. H. Fischer, M. Maksymenko, and E. Altman, Dynamics of a Many-Body-Localized System Coupled to a Bath, Phys. Rev. Lett. 116, 160401 (2016).

[32] H. P. Lüschen, P. Bordia, S. S. Hodgman, M. Schreiber, S. Sarkar, A. J. Daley, M. H. Fischer, E. Altman, I. Bloch, and U. Schneider, Signatures of Many-Body Localization in a Controlled Open Quantum System, Phys. Rev. X 7, 011034 (2017).

[33] T. Fukuhara, P. Schauß, M. Endres, S. Hild, M. Cheneau, I. Bloch, and C. Gross, Microscopic Observation of Magnon Bound States and Their Dynamics, Nature (London) 502, 76 (2013).

[34] See Supplemental Material at http://link.aps.org/ supplemental/10.1103/PhysRevX.9.041014 for more details.

[35] J. F. Sherson, C. Weitenberg, M. Endres, M. Cheneau, I. Bloch, and S. Kuhr, Single-Atom-Resolved Fluorescence Imaging of an Atomic Mott Insulator, Nature (London) 467, 68 (2010).

[36] T. B. Wahl, A. Pal, and S. H. Simon, Signatures of the Many-Body Localized Regime in Two Dimensions, Nat. Phys. 15, 164 (2019). 